\title{
Kapitel 2: Die Zirkularität theorieabhängiger empirischer Prüfungen
}

\section{Zirkularität durch Theoriebeladenheit}

Oft wird davon ausgegangen, dass die Theoriebeladenheit von Beobachtungen deshalb einen Pessimismus stützt, weil dadurch die empirische Prüfung wissenschaftlicher Theorien und Hypothesen zirkulär wird. Eine solche Zirkularität ist möglich, weil bei einer empirischen Prüfung durch theoriebeladene Beobachtung Theorien mindestens an zwei Stellen eine Rolle spielen. Zum einen gibt es mindestens eine Theorie, die durch die Beobachtung empirisch getestet werden soll, die zu prüfende Theorie. Zum anderen gibt es mindestens eine Theorie, von der die Beobachtung abhängt, die beladende Theorie. Es ist möglich, dass die zu prüfende Theorie mit der beladenden Theorie identisch ist oder zu den beladenden Theorien gehört. In diesen Fällen möchte ich von einer selbstabhängigen Prüfung sprechen. Dann übt die Theorie, die empirisch geprüft werden soll, einen Einfluss auf diejenige Instanz aus, mit der die Prüfung vorgenommen werden soll. Die Prüfung ist (partiell) zirkulär, und dies kann zweifelhaft machen, dass die Prüfung objektiv ist. ${ }^{1}$

Es gibt zwei vorherrschende Weisen, auf die solchermaßen genährten Bedenken aufgrund von Theoriebeladenheit zu reagieren. Zum einen wird darauf verwiesen, dass theoriebeladene Prüfungen nicht notwendig zirkulär sind. Oft wird eine Beobachtung nur durch Theorien beladen, die unabhängig von der zu prüfenden Theorie sind. Dann besteht keine Zirkularität. Viele Autoren schließen daraus, dass unter diesen Umständen trotz der Theoriebeladenheit von der Objektivität der Prüfung ausgegangen werden kann. ${ }^{2}$

Zum anderen wird darauf hingewiesen, dass auch die Beladung einer Beobachtung durch die zu prüfende Theorie oft nicht garantiert, dass das Beobachtungsergebnis die Theorie bestätigt. Vielmehr ist auch bei solcher Selbstabhängigkeit häufig ein Ergebnis möglich, das der Theorie widerspricht und sie daher empirisch untergräbt. Eine Reihe von Autoren hält theoriebela-

1 Vgl. Brown (1994), 406; Culp (1995), 439; Franklin et al. (1989); Kosso (1992), 154; Kosso/Kosso (1995), 583. Alle diese Autoren äußern zwar die Bedenken, sind aber der Ansicht, dass die Zweifel aus einem der Gründe, die im Folgenden diskutiert werden, letztlich unberechtigt sind.

2 Siehe Brown (1993), Carrier (2000), Franklin et al. (1989), Hacking (1983), 183-185, Kosso (1989) und (1992), Kosso/Kosso (1995). 
dene Beobachtungen, bei denen trotz der enthaltenen Zirkularität ein solches Fehlschlagsrisiko besteht, für objektiv. ${ }^{3}$

Die beiden Merkmale Unabhängigkeit und Fehlschlagsrisiko sind recht abstrakt, so dass sie auf Beobachtungen zutreffen können weitgehend unabhängig von den speziellen Arten der Beobachtungen und den besonderen Weisen ihrer Theoriebeladenheit. Man kann daher auf diese Merkmale in einer versuchten Begründung des Optimismus Bezug nehmen, die allgemein ist und nicht auf verschiedene Arten von Beobachtungen und Theoriebeladenheit eingeht. In dieser allgemeinen Weise werden sie auch häufig gegen einen Pessimismus aufgrund von Theoriebeladenheit angeführt, und diese Begründungen will ich an dieser Stelle - bevor Beobachtungsarten unterschieden und verschiedene Formen der Theoriebeladenheit genauer vorgestellt wurden, - untersuchen. Das Fehlschlagsrisiko durch theoriebeladene Beobachtungen werde ich in Abschnitt 2 diskutieren, die Unabhängigkeit von zu prüfender und beladender Theorie in Abschnitt 3.

Dabei wird sich zeigen, dass keines der beiden Merkmale in dieser abstrakten Form dazu hinreicht, um die Objektivität theoriebeladener Beobachtungen nachzuweisen. Zum einen wird sich ergeben, dass eine mögliche Zirkularität in der Prüfung gar nicht das zentrale epistemische Problem ist, das durch die Theoriebeladenheit von Beobachtungen entsteht. Zum anderen erweisen sich die Merkmale als solche als zu schwach, um die Probleme zu beheben.

Die im Folgenden zu besprechenden Autoren konzentrieren sich weitgehend auf die Rolle von Beobachtungen in der Prüfung (Bestätigung bzw. Widerlegung) von Theorien und Hypothesen und lassen andere mögliche epistemisch wichtige Rollen von Beobachtungen - etwa heuristische oder semantische - unberücksichtigt. Ich werde dieser Beschränkung folgen. Daher werde ich austauschbar von Beobachtung, Prüfung oder Test sprechen. Hierbei entscheidet sich die Objektivität von Beobachtungen daran, ob die empirische Bestätigung systematisch tugendhafter Theorien trotz der Theoriebeladenheit deren überwiegende Wahrheit wahrscheinlich macht. Nur dann darf man von der Bestätigung der Theorien auf deren Wahrheit schlieBen und damit gegenüber solchen Theorien optimistisch sein.

\section{Fehlschlagsrisiko und die Effektivität von Beobachtungen}

\section{a) Effektive und ineffektive Beobachtungen}

Die Merkmale Fehlschlagsrisiko und Unabhängigkeit schließen sich nicht aus. Eine empirische Prüfung kann sowohl nur von anderen Theorien als der zu

3 Diese Ansicht findet sich bei Brown (1993) und (1994), Carrier (1989) und (2000), Papineau (1979), 25, Shapere (1982), 516 (Fn. 17), Shogenji (2000). 
prüfenden Theorie beladen sein als auch für diese Theorie ein Fehlschlagsrisiko bergen. Allerdings wird das Merkmal Fehlschlagsrisiko in der Regel angeführt um zu zeigen, dass auch selbstabhängige Prüfungen objektiv sein können. So geht Harold Brown zunächst davon aus, dass unabhängig theoriebeladene Beobachtungen generell epistemisch nicht problematisch sind. Selbstabhängige Prüfungen sollen zudem ebenfalls objektiv sein, wenn die zu prüfende Theorie durch sie einem Fehlschlagsrisiko ausgesetzt wird. ${ }^{4}$

Das Verhältnis von Fehlschlagsrisiko und Objektivität lässt sich besonders gut an einem Beispiel untersuchen, das Brown präsentiert hat. ${ }^{5}$ Es geht um die Bestimmung der Geschwindigkeit, mit der zwei Quasare erstens sich von der Erde weg bewegen (Rezessionsgeschwindigkeit) und zweitens sich quer zur Sichtachse voneinander entfernen (Transversalgeschwindigkeit). Zur Bestimmung der Rezessionsgeschwindigkeit muss man zunächst die Rotverschiebung des Spektrums messen. Die Geschwindigkeit lässt sich dann berechnen, wenn man davon ausgeht, dass die Verschiebung des Spektrums auf dem Dopplereffekt beruht. Hierzu verwendet man die Formel für den Dopplereffekt, die sich aus der Speziellen Relativitätstheorie ergibt:

(SRT-Doppler)

$$
\beta=\frac{S^{2}-1}{S^{2}+1} \text {. }
$$

Hierbei steht $S$ für das Verhältnis von verschobener zu unverschobener Wellenlänge, $\beta$ bezeichnet das Verhältnis von Rezessionsgeschwindigkeit zu Lichtgeschwindigkeit.

Man sieht, dass keine Werte für die Rezessionsgeschwindigkeit möglich sind, die größer oder gleich der Lichtgeschwindigkeit sind, da $\beta$ nicht größer oder gleich 1 sein kann. Es ist somit kein Ergebnis möglich, dass der Konsequenz der Speziellen Relativitätstheorie widersprechen würde, dass Quasare sich langsamer als mit Lichtgeschwindigkeit von der Erde entfernen.

Um die Transversalgeschwindigkeit zu bestimmen, misst man zunächst, wie schnell sich der Abstand zwischen den beiden Quasaren, von der Erde aus gesehen, vergrößert (Winkelgeschwindigkeit). Aus der Rezessionsgeschwindigkeit und der Hubble-Konstante lässt sich zudem der Abstand der Quasare zur Erde berechnen. Aus Abstand und Winkelgeschwindigkeit ergibt sich dann die Transversalgeschwindigkeit. Von besonderem Interesse ist, dass die Werte für die Transversalgeschwindigkeit sehr wohl größer als Lichtgeschwindigkeit ausfallen können, obwohl man auch hier (durch die Verwendung der berechneten Rezessionsgeschwindigkeiten) die Spezielle Relativi-

\footnotetext{
4 Siehe Brown (1993).

5 Für das Folgende und weitere Details siehe Brown (1993), 555-557.
} 
tätstheorie voraussetzt. Solche Ergebnisse, die der Relativitätstheorie prima facie widersprechen, hat man im Laufe der Forschungen tatsächlich erhalten.

In diesem Fallbeispiel kommen damit zwei Beobachtungen vor, die plausiblerweise von sehr verschiedenem epistemischen Wert sind. Die Beobachtung der Rezessionsgeschwindigkeit ist für die Spezielle Relativitätstheorie evidenziell irrelevant. Die Werte, die man für die Rezessionsgeschwindigkeit erhält, sollten keinen Einfluss auf den Umstand haben, ob man die Relativitätstheorie für wahr hält. Die Beobachtung kann also die Theorie nicht bestätigen oder untergraben. Das Ergebnis für die Transversalgeschwindigkeiten kann dagegen sehr wohl einen Einfluss auf die Einschätzung der Relativitätstheorie haben. Ich will zur Benennung dieses Unterschieds zunächst einen neuen Ausdruck einführen, um der Diskussion nicht vorzugreifen, wie der Unterschied genau zu analysieren ist. Daher nenne ich ein empirisches Ergebnis wie das erste, das keine mögliche Bestätigungsrelevanz für die fragliche Theorie hat, ineffektiv. Ein Beobachtungsbefund wie das zweite Ergebnis, das sehr wohl eine solche Relevanz für eine Theorie haben kann, ist dagegen für diese Theorie effektiv ${ }^{6}$

Brown behauptet, dass der epistemisch zentrale Unterschied zwischen den beiden Fällen darin besteht, ob die zu prüfende Theorie durch das Beobachtungsergebnis einem Fehlschlagsrisiko ausgesetzt ist. In der eben eingeführten Terminologie ausgedrückt hängt Brown zufolge die Effektivität selbstabhängig theoriebeladener Beobachtungen daran, dass für die zu prüfende Theorie ein Fehlschlagsrisiko besteht. Nun gibt es zwischen den Fällen zwar einen Unterschied hinsichtlich des Fehlschlagsrisikos. Man kann aber überlegen, ob es wirklich dieser Unterschied ist, der für die - unstrittige - Differenz im epistemischen Wert der Fälle verantwortlich ist.

Brown behauptet zudem, dass das Bestehen eines Fehlschlagsriskos ausreicht, um erkenntnistheoretische Bedenken aufgrund der Theoriebeladenheit auch bei zirkulären Prüfungen zu zersteuen. Er schreibt:

We have seen that the theory-dependence of observation can block a particular observational test. ... We have also seen that an observation that cannot itself test a specific claim of a theory can still play a role in a more complex procedure that can test that very claim. ... [The] familiar claim that an observation that depends in an essential way on a theory cannot provide an objective test of that very theory is false. (Brown 1993, 558/559; Browns Hervorhebungen.)

6 Brown (1994) selbst spricht nicht von Effektivität, sondern davon, ob eine Prüfung einen echten Test („fair empirical test") darstellt. Carrier (2000) spricht von ,,aussagekräftigen“" Prüfungen. Den Ausdruck ,Effektivität' habe ich von Shogenji (2000), 297 übernommen. 
$[T]$ heory-dependence does not undermine the objectivity of the evidence derived from an empirical procedure because even the most heavily theory-dependent procedures can yield unexpected and unwanted empirical outcomes. (Brown 1995, 394)

Wie Brown selbst schreibt, sind beide Behauptungen Ausdruck einer „quasiPopper'schen“ Auffassung der Bestätigungsrelevanz empirischer Prüfungen. Zum einen wird gefordert, dass empirische Prüfungen zu einem Resultat führen können, das die Theorie empirisch untergräbt. Zum anderen wird aber zugestanden, dass mit solchen Tests die Theorie objektiv bestätigt werden kann. ${ }^{7}$

Hier stellen sich zwei Fragen. Erstens ist zu untersuchen, ob Brown den zentralen Unterschied zwischen den beiden Beobachtungsergebnissen korrekt analysiert und die Effektivität selbstabhängiger Prüfungen tatsächlich vom Fehlschlagsrisiko abhängt. Zweitens muss man fragen, ob die Effektivität von Beobachtungen wirklich ausreicht, um ihre Objektivität angesichts von Theoriebeladenheit zu begründen. Den beiden Fragen werde ich in den folgenden beiden Unterabschnitten nachgehen.

\section{b) Wovon hängt die Effektivität von Beobachtungen ab?}

Tomoji Shogenji hat sich gegen Browns Analyse der Effektivität in den Beispielen gewandt. Er hat behauptet, dass die Effektivität von Beobachtungen nicht vom Fehlschlagsrisiko abhängt, sondern davon, ob die Prüfhypothese für die Vorhersage bzw. Ableitung des Beobachtungsresultats relevant ist. Shogenji formuliert diese Behauptungen in Begriffen der Bayesianischen Bestätigungstheorie. Um die Thesen und ihre Begründung präzise rekonstruieren zu können, ist es nützlich, sich hierin zunächst Shogenji anzuschließen.

Ein Beobachtungsergebnis $O$ ist - bayesianisch ausgedrückt - für eine Prüfhypothese $H$ gerade dann ineffektiv, wenn das Erhalten des Beobachtungsergebnisses die (subjektive) Wahrscheinlichkeit der Hypothese unverändert lässt. Umgekehrt ist eine Beobachtung genau dann effektiv, wenn sie die Wahrscheinlichkeit der Hypothese erhöht oder verringert. Es gilt:

(Eff) $O$ ist effektiv genau dann, wenn $O$ dazu führt, dass $P^{\prime}(H) \neq P(H)$.

$\left(P^{\prime}(H)\right.$ bezeichnet hierbei die Hypothesenwahrscheinlichkeit nach der Beobachtung, $P(H)$ die Wahrscheinlichkeit vor der Beobachtung.)

Die Prüfung und Bestätigung von Hypothesen spielt sich in der Regel vor dem Hintergrund weiterer Annahmen ab, die auch für die Bayesianische

7 Siehe Brown (1994), 409. Vgl. auch Shogenji (2000), 292, der eine Bayesianische Begründung dieser Auffassung gibt. 
Beschreibung wichtig sind. Shogenji bezeichnet mit $B^{*}$ die relevanten Hintergrundannahmen, wobei der Stern andeuten soll, dass hierin die Prüfhypothese $H$ nicht enthalten ist. Im obigen Satz (Eff) sind dann eigentlich bedingte Wahrscheinlichkeiten $P\left(H / B^{*}\right)$ und $P^{\prime}\left(H / B^{*}\right)$ zu lesen.

Shogenji zufolge soll die Ineffektivität theorieabhängiger Prüfungen darauf beruhen, dass der reduzierte Hintergrund $B^{*}$ allein die Beobachtung $O$ in gleichem Maß erwarten lässt wie $B^{*}$ und $H$ zusammen. Seine Analyse sieht also vor, dass eine theoriebeladene Prüfung ineffektiv ist, weil die Prüfhypothese $H$, gegeben den Hintergrund $B^{*}$, prädiktiv irrelevant ist:

(Präd-Irrel)

$$
P\left(\mathrm{O} / B^{*}\right)=P\left(\mathrm{O} / B^{*} \& H\right) \text {. }
$$

Auch die Ineffektivität der Rezessionsgeschwindigkeiten für die Spezielle Relativitätstheorie soll sich Shogenji zufolge so erklären lassen. ${ }^{8}$

Was ist von diesem Vorschlag zu halten? Zunächst muss man festhalten, dass die prädiktive Irrelevanz einer Hypothese tatsächlich dazu führt, dass eine Prüfung die Hypothesenwahrscheinlichkeit unverändert lässt. Denn wenn die Hypothese prädiktiv irrelevant ist, ergibt sich mit Bayes’ Theorem

$$
P\left(H / B^{*} \& O\right)=P\left(H / B^{*}\right) \cdot \frac{P\left(O / B^{*} \& H\right)}{P\left(O / B^{*}\right)},
$$

dass gilt:

$$
P\left(H / B^{*} \& O\right)=P\left(H / B^{*}\right) .
$$

Wenn man nun die Standardregel für Konditionalisierung voraussetzt, wie Shogenji es tut, dass nämlich die Hypothesenwahrscheinlichkeit nach der Beobachtung gleich der bedingten Wahrscheinlichkeit vor der Beobachtung ist, also

(Stand-Kond)

$$
P^{\prime}\left(H / B^{*}\right)=P\left(H / B^{*} \& O\right),
$$

dann folgt, dass die Beobachtung für die Hypothese ineffektiv ist:

$$
P^{\prime}\left(H / B^{*}\right)=P\left(H / B^{*}\right)^{9}
$$

Allerdings ergibt sich bei genauerem Hinsehen, dass diese Analyse nicht ohne Weiteres die Ineffektivität der Beobachtung der Rezessionsgeschwindigkeiten in Browns Beispiel erklären kann. Shogenjis Analyse setzt mit (PrädIrrel) voraus, dass das beobachtete Resultat allein vor dem Hintergrund $B^{*}$ genauso erwartbar war wie unter der zusätzlichen Voraussetzung der Prüfhypothese $H$. In Browns Beispiel wäre $H$ als Spezielle Relativitätstheorie

\footnotetext{
8 Siehe Shogenji (2000), 296/297.

9 Vgl. Shogenji (2000), 288/289.
} 
aufzufassen und $B^{*}$ als das um diese Theorie reduzierte Hintergrundwissen. Die Beobachtung $O$, dass alle Quasare sich unter Lichtgeschwindigkeit entfernen, erscheint aber allein vor diesem reduzierten Hintergrund, der die Spezielle Relativitätstheorie nicht enthält, viel weniger wahrscheinlich, als bei zusätzlicher Annahme der Relativitätstheorie. Denn die Relativitätstheorie impliziert das Beobachtungsergebnis, es ist $P\left(O / B^{*} \& H\right)=1$, der reduzierte Hintergrund impliziert dies nicht, es ist $P\left(O / B^{*}\right)<1$. Daher ist in diesem Fall gerade die Relativitätstheorie für die Vorhersage des Ergebnisses relevant, (Präd-Irrel) ist nicht erfüllt.

Der Defekt in Shogenjis Rekonstruktion lässt sich genauer lokalisieren und damit eine möglicherweise rettende Revision vorschlagen, wenn man die dahinter stehende Idee expliziert. ${ }^{10}$ Bisher haben wir der zu prüfenden Hypothese $H$ zwei verschiedene Rollen in der Prüfung zugeschrieben. Erstens wird $H$ herangezogen, um zusammen mit dem reduzierten Hintergrund $B^{*}$ die Beobachtung $O$ vorherzusagen:
1. $H$ in der Vorhersage:
$H \& B^{*} \rightarrow O$.

Zweitens trägt $H$ dazu bei, das Beobachtungsergebnis empirisch zu gewinnen. Der Beobachtungsprozess führt demnach mit Hilfe von $H$ über verschiedene Stadien, eventuell über ein Zwischenergebnis $O^{*}$ und unter Inanspruchnahme von Bestandteilen des Hintergrundwissens $B^{*}$, zu $O$ :

\section{2. $H$ im Beobachtungsprozess: $\quad O * \& H \& B^{*} \quad O$.}

Shogenjis Idee ist nun, dass man dieselbe Prüfung auch so beschreiben kann, dass $H$ nur in einer einzigen Rolle auftritt, nämlich in der Vorhersage eines Beobachtungsergebnisses. Dies ist aber offenkundig nur möglich, wenn nicht $O$ vorhergesagt wird. Denn es wird gerade angenommen, dass $H$ für das Zustandkommen von $O$ notwendig ist. In Browns Beispiel kann man die Rezessionsgeschwindigkeiten nur empirisch bestimmen, wenn man eine Hypothese über den Dopplereffekt bei Licht verwendet. Ohne die Annahme des relativistischen Dopplereffekts oder einer Ersatzhypothese ist $O$ also gar nicht zu bekommen.

Es ist jedoch denkbar, dass Shogenjis Vorschlag in modifizierter Weise erfolgreich ist. Die Rolle von $H$ in der Gewinnung des Beobachtungsergebnisses ist eliminierbar, wenn statt des Ergebnisses $O$ ein Zwischenergebnis $O^{*}$ vorhergesagt werden kann, für dessen empirische Gewinnung $H$ nicht benötigt wird. Dann würde die modifizierte Vorhersage wie folgt aussehen:

1 .

$$
H \& B^{*} \rightarrow O^{*}
$$

10 Für das Folgende vgl. Shogenji (2000), 292/293. 
Die Gewinnung des vorhergesagten Ergebnisses würde die Hypothese nicht mehr verwenden müssen:

2'. $\mathrm{O}^{*}$.

In Browns Beispiel scheint es möglich, eine solche Zwischenstufe anzugeben, nämlich die festgestellten Verhältnisse von verschobener zu unverschobener Wellenlänge $S$. Man kann dieses Verhältnis wohl empirisch bestimmen, ohne die Spezielle Relativitätstheorie zu verwenden. Gleichzeitig lässt es sich aus der Speziellen Relativitätstheorie ableiten. Das Verhältnis der Wellenlängen stellt dann ein Zwischenergebnis der gesuchten Art $O^{*}$ dar.

Bei dieser Rekonstruktion ist zudem Shogenjis Erklärung der Ineffektivität plausibel. Denn die Spezielle Relativitätstheorie wird zwar zweimal verwendet, um die möglichen Werte für $S$ zu berechnen. Erstens folgt aus ihr, dass das Verhältnis $\beta$ von Rezessionsgeschwindigkeit zu Lichtgeschwindigkeit kleiner als eins ist. Daraus erhält man eine Vorhersage für $S$, indem man zweitens den relativistischen Dopplereffekt (SRT-Doppler) annimmt. Aber diese Vorhersage ist vollkommen leer: Wenn $\beta$ kleiner als eins ist, kann der obigen Gleichung (SRT-Doppler) zufolge $S$ jeden beliebigen Wert annehmen. Die Voraussetzung der Speziellen Relativitätstheorie erlegt den erwarteten Werten für $S$ gar keine über den Hintergrund $B^{*}$ hinausgehenden Einschränkungen auf. Die Annahme der Speziellen Relativitätstheorie ist in diesem Fall irrelevant dafür, welche Beobachtungen man erwartet.

Die revidierte Analyse von Shogenji erweist sich damit als durchaus fähig, den epistemischen Unterschied zwischen den beiden Fällen zu erklären. ${ }^{11}$ Bei den Transversalgeschwindigkeiten können sich aus der Speziellen Relativitätstheorie Beschränkungen für die gemessenen Winkelgeschwindigkeiten ergeben. Im Fall der Rezessionsgeschwindigkeiten führt die zusätzliche Annahme der Speziellen Relativitätstheorie dagegen zu keiner Einschränkung der erwartbaren Rotverschiebungen, die über den weiteren Hintergrund hinausgehen. Die skizzierte einfache bayesianische Argumentation zeigt dann, warum die Beobachtungen nur im einen Fall, nicht aber im anderen die Hypothesenwahrscheinlichkeit beeinflussen können.

Allerdings hat die Analyse einen entscheidenden Nachteil. Sie erklärt primär nicht die Ineffektivität von $O$, sondern die der Vorstufe $O^{*}$. Daher ist sie darauf angewiesen, dass es eine geeignete Vorstufe $O^{*}$ auch gibt. Diese

11 Die Analyse ist gegenüber Shogenjis eigenem Vorschlag insofern revidiert, als es nicht darum geht, das ursprüngliche Ergebnis $O$, sondern ein zugrunde liegendes Zwischenergebnis $O^{*}$ vorherzusagen. Wie gezeigt, führt nur der revidierte Vorschlag dazu, die Rolle von $H$ auf die Vorhersage zu beschränken, wenn die Gewinnung von $O$ auf $H$ angewiesen ist. 
Vorstufe muss zum einen durch $H$ und den Hintergrund $B^{*}$ vorhersagbar sein. Zum anderen darf sie selbst nicht von $H$ abhängen. Damit ist Shogenji aber darauf angewiesen, dass die Beladung eines empirischen Ergebnisses $O$ durch eine zu prüfende Theorie sich immer eliminieren lässt, indem man auf ein zugrunde liegendes, insoweit theorieunabhängiges Ergebnis $O^{*}$ zurückgeht. Nur dann lässt sich das Problem, das zunächst an der Theorieabhängigkeit der empirischen Basis $O$ zu hängen scheint, als ein Problem rekonstruieren, das am Verhältnis zwischen neutraler Basis $O^{*}$ und Hypothese $H$ hängt. In den folgenden Kapiteln werden aber überwiegend Formen der Theoriebeladenheit zu besprechen sein, bei denen ein solcher Rückgriff auf ein zugrunde liegendes, nicht theoriebeladenes Beobachtungsergebnis nicht sinnvoll möglich ist. Diesen Formen zufolge braucht man Theorien, um überhaupt erst ein sinn- bzw. gehaltvolles empirisches Ergebnis zu erhalten, oder um ein solches Ergebnis zuerst als verlässlich einschätzen zu können. Es macht daher Sinn, an dieser Stelle nach einer Analyse von effektiver und ineffektiver Beobachtung zu suchen, die nicht auf zugrunde liegende theorieunabhängige Beobachtungen angewiesen ist.

Für eine solche Analyse hat die Diskussion von Shogenji allerdings schon ein gutes Stück weit den Weg gewiesen. Denn wenn man diese Analyse abstrakter betrachtet, hat sie gezeigt, dass sich die Beobachtungen in Browns Beispiel darin unterscheiden, ob in ihnen überhaupt empirische Beiträge vorkommen, die für die Spezielle Relativitätstheorie relevant sind. Dies kann anhand der eingeführten Vorstufe $O^{*}$ deutlich gemacht werden. Diese Vorstufe ist von der Relativitätstheorie unabhängig. Wir können für die jetztigen Zwecke davon ausgehen, dass das Ergebnis völlig theorieunabhängig ist und daher vollständig empirisch bestimmt wird. Das Ergebnis $O$ erhält man dann durch einen Rechenschritt, in den neben $O^{*}$ allein theoretische Annahmen einfließen. In diesem Schritt kommen daher keine empirischen Beiträge hinzu; soweit $O$ empirisch bestimmt ist, wird dies vollständig durch $O^{*}$ vermittelt. $O^{*}$ enthält daher alle empirischen Beiträge, die $O$ enthalten kann.

Die prädiktive Irrelevanz von $H$ für $O^{*}$ zeigt zunächst, dass $O^{*}$ gar keine Gründe für oder gegen $H$ liefert. $O$ enthält aber nichts Empirisches, das nicht in $O^{*}$ enthalten ist. Daher zeigt die prädiktive Irrelevanz von $H$ für $O^{*}$ auch, dass $O$, soweit es empirisch bestimmt ist, gar keine Gründe für oder gegen $H$ liefern kann. $O$ ist zwar seinem Inhalt nach mit $H$ vereinbar und scheint dieses daher zunächst zu stützen. Diese Stützung beruht aber nicht auf empirischen Gründen, die $O$ für $H$ liefern würde. $O$ fällt unabhängig davon, wie die Welt tatsächlich beschaffen ist und allein wegen der theoretischen Bestimmung im Schritt von $O^{*}$ zu $O$ für $H$ günstig aus. Dieser Analyse zufolge ist $O$ also für 
$H$ ineffektiv, weil das Ergebnis, soweit es empirisch bestimmt ist, gar nichts für oder gegen $H$ besagen kann.

In dem Umfang, in dem $O$ theoretisch und nicht empirisch bestimmt ist, kann es für keine damit geprüfte Theorie effektiv sein. Im Fall der Speziellen Relativitätstheorie führt der nicht-empirische Charakter von $O$ dazu, dass $O$ für die Relativitätstheorie kein Fehlschlagsrisiko birgt. Bei anderen Theorien kann die Ineffektivität sich aber auch darin zeigen, dass es mit den Theorien unvereinbar ist unabhängig davon, wie die Welt beschaffen ist. Solche Theorien haben gar keine Chance, durch ein Ergebnis bestätigt zu werden, das man auf die gleiche Weise wie $O$ erhält.

Dies lässt sich an einem konstruierten Beispiel zeigen. Nehmen wir an, wir wollen mit dem Beobachtungsergebnis eine "Turboquasar-Theorie“ testen, derzufolge Quasare sich sehr wohl schneller als Licht entfernen können. Die berechneten Ergebnisse scheinen diese Theorie zunächst zu untergraben. Wenn es tatsächlich solche Turboquasare gibt, sollten wir sie doch finden können? Ein genauerer Blick auf die Genese der Beobachtungsergebnisse zeigt aber, dass es gar nicht möglich ist, durch das gegebene Vorgehen solche Geschwindigkeiten zu erhalten. Egal wie schnell sich Quasare bewegen und wie sehr ihre Spektren daher verschoben sind: Unsere Weise der Berechnung ihrer Geschwindigkeit allein bestimmt, dass die Ergebnisse kleiner als Lichtgeschwindigkeit ausfallen. Da das Beobachtungsergebnis hinsichtlich der Frage, ob es Quasare gibt, die schneller als Licht sind, gar nicht empirisch ist, zeigt der Widerspruch mit der Turboquasar-Theorie lediglich, dass diese Theorie mit der Speziellen Relativitätstheorie unvereinbar ist. Das mag zwar ein starker Grund sein, die Turboquasar-Theorie aufzugeben. Es ist aber kein empirischer Grund, der sich aus der gegebenen Beobachtung ergibt. Daher liefert die Beobachtung auch keine empirischen Gründe für oder gegen die Turboquasar-Theorie, und sie ist auch für diese Theorie ineffektiv. ${ }^{12}$

Die berechneten Quasar-Geschwindigkeiten sind natürlich hierbei nur in bestimmter Hinsicht theoretisch vollständig festgelegt, nämlich dahingehend, dass die Geschwindigkeiten kleiner als die Lichtgeschwindigkeit sind. Der genaue Wert der Rezessionsgeschwindigkeit hängt dagegen wiederum von der gemessenen Rotverschiebung ab und ist damit auch empirisch mitbestimmt. Allerdings ist das Ergebnis nur in der Hinsicht, in der es vollständig theoretisch determiniert ist, direkt für die Spezielle Relativitätstheorie und die

12 Vgl. auch Brown (1995), 368, wo er die epistemologische Unbedenklichkeit einer Form der Theoriebeladenheit ebenfalls damit begründet, dass die Theorie das Beobachtungsergebnis nicht vollständig festlege. Allerdings findet sich bei Brown keine systematische Ausarbeitung dieses Kriteriums oder ein Vergleich mit dem sonst immer angeführten Merkmal „Fehlschlagsrisiko“. 
Turboquasar-Theorie relevant. Dieses Teilergebnis wird gerade durch die Beobachtungsproposition $O$ erfasst, die daher ineffektiv ist.

Das Ergebnis für die Transversalgeschwindigkeiten ist demgegenüber für beide Theorien effektiv. Dies liegt zum einen daran, dass hier nicht nur $O$ einfließt, sondern die genauen Werte für die Rezessionsgeschwindigkeiten. Daraus berechnet sich der Abstand der Quasare von der Erde. Zum anderen fließen zusätzliche empirische Informationen in Form der gemessenen Winkelgeschwindigkeiten ein. Daher ist der Ausgang der Beobachtung der Transversalgeschwindigkeiten für beide Theorien davon abhängig, wie die Welt tatsächlich beschaffen ist. Beide Theorien haben eine Chance, empirisch bestätigt zu werden, und beide setzen sich der Gefahr eines empirischen Fehlschlags aus.

Diese Analyse der Effektivität und Ineffektivität lässt sich ebenfalls in bayesianischen Begriffen ausdrücken. Demnach kann es wegen Theoriebeladenheit vorkommen, dass man ein Beobachtungsergebnis $O$ erhält, ohne damit einen empirischen Grund dafür zu haben, den Überzeugungsgrad von $O$ zu verändern. In einem solchen Fall wäre, obwohl man $O$,beobachtet' hat,

(Nicht-Emp)

$$
P^{\prime}(O)=P(O) \cdot{ }^{13}
$$

(Der Einfachheit halber verzichte ich hier und im Folgenden auf die Bedingtheit durch den Hintergrund $B^{*}$, die bei allen Wahrscheinlichkeiten eigentlich mitzuschreiben wäre.)

Die Ineffektivität nicht-empirischer Beobachtungen lässt sich nun bayesianisch nicht allgemein beschreiben, solange man nur von der Standardregel für Konditionalisierung (Stand-Kond) ausgeht. Denn diese Regel ist nur anwendbar, wenn die Beobachtungsproposition nach der Beobachtung die Wahrscheinlichkeit eins hat, und dies ist bei nicht-empirischen Beobachtungen in der Regel nicht gegeben. Richard Jeffrey hat aber die folgende Verallgemeinerung von (Stand-Kond) vorgeschlagen, die auch für Fälle gilt, in denen die

13 Die Annahme, dass die Wahrscheinlichkeit von $O$ unverändert bleibt, wenn das Zustandekommen dieses Ergebnisses nicht von empirischen Umständen abhängt, muss man hinzunehmen, um eine bayesianische Beschreibung der Ineffektivität aufgrund von Theoriebeladenheit zu ermöglichen. Diese Annahme folgt aber nicht aus den sonstigen Annahmen des Bayesianismus oder der Wahrscheinlichkeitstheorie. Der Bayesianismus kann insofern die Ineffektivität von $O$ nicht wirklich erklären, sondern nur beschreiben. Dies liegt daran, dass wahrscheinlichkeitstheoretisch nur Beziehungen zwischen Propositionen betrachtet werden. Demgegenüber liegt der Grund der Ineffektivität von $O$ gerade in der Weise, in der das Ergebnis zustande kommt. Wenn diese Genese keine geeignete Vorstufe $O^{*}$ einschließt, kann sie rein bayesianisch nicht in den Blick genommen werden. 
Beobachtungsproposition nach der Beobachtung nicht die Wahrscheinlichkeit eins hat:

$$
\text { (Jeffrey-Kond) } \quad P^{\prime}(H)=P(H / O) \cdot P^{\prime}(O)+P(H / \sim O) \cdot P^{\prime}(\sim O){ }^{14}
$$

Wenn man diese Regel voraussetzt, ergibt sich, dass eine Prüfung ineffektiv ist genau dann, wenn die Prüfung nicht-empirisch oder die Prüfhypothese prädiktiv irrelevant ist, d.h.

(Ineff)

$$
P^{\prime}(H)=P(H) \text { g.w.d. } P^{\prime}(O)=P(O) \text { oder } P(O / H)=P(O) .
$$

(Das „oder“ ist inklusiv. Für die Ableitung von Ineff siehe den Anhang zu diesem Kapitel).

Die Bayesianische Rekonstruktion ergibt also, dass die Ineffektivität einer empirischen Prüfung auf zwei Umständen beruhen kann. Zum einen kann die Testhypothese prädiktiv irrelevant sein. Auf diese Möglichkeit hat Shogenji hingewiesen. Eine Beobachtung ist dann aber nicht wegen ibrer Theorieabhängigkeit ineffektiv. Wie gesehen, bietet sich diese Erklärung der Ineffektivität bei Theoriebeladenheit nur an, wenn es eine theorieunabhängige Vorstufe gibt. Erklärt wird zudem dann die Ineffektivität der Vorstufe und nicht die des theoriebeladenen empirischen Ergebnisses.

Zum anderen kann eine Beobachtung nicht-empirisch sein, d.h. gar keine empirischen Gründe für oder gegen die Beobachtungsproposition geben. Dies kommt dann vor, wenn das Beobachtungsergebnis in der Hinsicht, die durch die Beobachtungsproposition beschrieben wird, durch die Theoriebeladung vollständig festgelegt ist.

\section{c) Effektivität und Objektivität}

Sind effektive Beobachtungen trotz ihrer Theoriebeladenheit objektiv? Aus dem Zitat in Abschnitt 2a geht hervor, dass Brown dieser Ansicht zu sein scheint, und auch andere Autoren haben ähnliche Ansichten geäußert. ${ }^{15}$ Man darf hierbei natürlich nicht ohne Weiteres davon ausgehen, dass sie die Objektivität von Beobachtungen im Sinn meiner Zielvorgabe verstehen, derzufolge die Beschaffenheit von Beobachtungen eine partielle Begründung des Optimismus ermöglichen soll. Daher ist nicht vollkommen klar, dass sie behaupten, effektive Beobachtungen seien im Sinn der Zielvorgabe objektiv. Allerdings ist die Unterstellung einer solchen Behauptung auch nicht völlig unberechtigt. Denn die genannten Autoren diskutieren die Effektivität von Beobachtungen im Zusammenhang mit der Frage, ob der besondere epis-

14 Jeffrey (1967), 209. Vgl. hierzu auch Howson/ Urbach (1989), $284 \mathrm{ff}$.

15 Vgl. etwa Shapere (1982), 516 (Fn. 17). 
temische Status wissenschaftlicher Erkenntnisse durch die Theoriebeladenheit in Frage gestellt werde. Die Zurückweisung des Pessimismus wird dann damit begründet, dass die Theoriebeladenheit auch bei selbstabhängigen Beobachtungen nicht immer zu Ineffektivität führt.

Wenn effektive Beobachtungen objektiv sind, müssen sich hierdurch trotz der Theoriebeladenheit die guten Erkenntnisaussichten der Wissenschaften begründen lassen. Die Gefahr für den epistemischen Erfolg besteht hier darin, dass überwiegend falsche Theorien empirisch bestätigt werden können, weil die Beobachtungen theoretisch beeinflusst werden. Wenn Beobachtungen ineffektiv sind, ist diese Gefahr besonders groß. Denn dann werden die Beobachtungsergebnisse, zumindest soweit sie für die Hypothesenprüfung relevant sind, vollständig von Theorien festgelegt. Es hängt gar nicht von der Beschaffenheit der Welt ab, welche Beobachtungsergebnisse man erhält.

Wenn Theoriebeladenheit zu weitreichender Ineffektivität der Beobachtungen führen würden, wäre in der Tat der epistemische Erfolg der Wissenschaften höchst zweifelhaft. Diese Zweifel bestünden weitgehend unabhängig davon, ob es jeweils dieselben oder verschiedene Theorien wären, die die Beobachtungsergebnisse festlegen. Zwar könnte man argumentieren, dass falsche Theorien durch selbstabhängige Prüfungen noch eher bestätigt würden. (Hierzu gleich mehr im nächsten Abschnitt.) Aber ebenso gut könnten weitgehend falsche Theorien durch Beobachtungen, die von unabhängigen falschen Theorien festgelegt werden, bestätigt werden. Das Problem ineffektiver Beobachtungen ist daher keine Folge nur einer möglichen Zirkularität.

Gegenüber dieser pessimistischen Argumentation ist es wichtig, dass Beobachtungen in der Regel trotz ihrer Theoriebeladenheit effektiv sind. Daher ist die Effektivität ein wichtiges Merkmal objektiver Beobachtungen. Allerdings ist es bestenfalls eine minimale Bedingung für objektive Beobachtungen. Es ist damit nur gesagt, dass es auch von der Welt abhängt, welches empirische Ergebnis man erhält. Es ist erstens nicht gesagt, wie stark der Einfluss der Welt ist. Beobachtungen sind schon effektiv, wenn ein Ergebnis unter allen denkbaren Beschaffenheiten des Objekts außer unter sehr ungewöhnlichen immer so ausfällt, wie von der beladenden Theorie bestimmt. Dagegen müssten Beobachtungsergebnisse, die epistemisch wirklich wertvoll sind, sehr präzise mit verschiedenen Objektzuständen variieren. Zweitens ist durch die Effektivität von Beobachtungen gar nichts über ihre Wahrheit oder Falschheit gesagt. Beobachtungen können sowohl effektiv als auch immer irreführend sein, etwa weil falsche Theorien sie beladen. Dann kann aber allein unter Bezugnahme auf die Effektivität von Beobachtungen keine Annahme über die Wahrscheinlichkeit der Wahrheit von damit bestätigten 
Theorien begründet werden. Die Objektivität von Beobachtungen ist allein durch ihre Effektivität nicht zu begründen.

\section{Sind unabhängig theoriebeladene Beobachtungen objektiv?}

a) Unabhängig theoriebeladene Beobachtungen

Viele Autoren sind der Ansicht, dass die Objektivität theoriebeladener Beobachtungen bzw. einer mit ihnen vorgenommenen empirischen Prüfung jedenfalls dann gewährleistet ist, wenn geprüfte Theorie und beladende Theorie voneinander unabhängig sind. So schreibt etwa Peter Kosso:

With the requirement of independence we can ease what may have appeared to be a tension between two key components in the description of science, the facts that observations are influenced by theory and that these observations are the evidence used to test theories. This relationship need not be circular as the theory that does the influencing need not be the same as the one that takes the test. This circleblocking independence is a measure of objectivity of the evidence and of the process of justification. (Kosso 1992, 157/158)

All evidence in science bears the mark of theory, but it does not have to be the mark of the particular theory being tested. Insuring that it is not is the accomplishment of objectivity in justification. (Kosso 1992, 161)

Hier wird behauptet, dass die Unabhängigkeit von beladender und geprüfter Theorie zur Objektivität der Beobachtungen beiträgt. Dabei steht im ersten Zitat der Vergleich zu zirkulären, d.h. selbstabhängigen Beobachtungen im Vordergrund. Kossos These ist hierbei komparativ: Beobachtungen sind objektiver, wenn beladende und geprüfte Theorie voneinander unabhängig sind. ${ }^{16}$ Im zweiten Zitat scheint Kosso dagegen zu behaupten, dass Unabhängigkeit angesichts der Theoriebeladenheit die Objektivität sicherstellt. Beide Thesen sind im Folgenden von Interesse. Wenn man davon ausgeht, dass bei Selbstabhängigkeit nicht generell gesichert ist, dass Beobachtungen objektiv sind, sieht die erste, komparative These zumindest eine Verbesserung der epistemischen Qualität bei unabhängiger Beladung vor. Damit ist aber noch

16 Kosso hat diese Position ausgearbeitet, indem er verschiedene Stufen der Unabhängigkeit von Theorien (bzw. Theorieteilen) unterscheidet, und zwar in Abhängigkeit davon, in welchem Ausmaß die Theorien überlappen oder die Wahrheit der einen Theorie für oder gegen die Wahrheit von Propositionen der anderen Theorie spricht. Zudem behauptet er, dass theoriebeladene Beobachtungen umso objektiver sind, je unabhängiger zu prüfende Theorie und beladende Theorie sind. Siehe hierzu Kosso (1989). Da meine Kritik am Kriterium „Unabhängigkeit“ sehr grundsätzlich ausfallen wird, werde ich diese Ausarbeitung vernachlässigen und nur die vereinfachte These behandeln, dass Tests, die zu welchem Grad auch immer unabhängig sind, objektiver sind als entsprechende selbstabhängige Prüfungen. 
nicht klar, ob die Verbesserung soweit geht, dass Unabhängigkeit für Optimismus ausreicht. Dies behauptet erst die zweite These.

Die obige Diskussion der Effektivität von Beobachtungen hat allerdings gezeigt, dass auch unabhängig theoriebeladene Beobachtungen ineffektiv sein können. Effektivität ist aber eine minimale Bedingung für objektive Beobachtungen. Es scheint daher am sinnvollsten, Kosso so zu verstehen, dass er behauptet, unabhängige und effektive Beobachtungen seien objektiv. Die zweite, in diesem Abschnitt zur Diskussion stehende These soll daher sein, dass Unabhängigkeit zusammen mit Effektivität hinreichend für Objektivität ist.

Das folgende Zitat deutet die Überlegungen an, auf die sich Kosso in der Begründung dieser These stützt:

[Why is the] feature of independence between accounting theories and the theory being tested a truth-conducive feature? The goal of validation of scientific knowledge is the elimination of theoretical artifacts. We want the entities and events described by a natural science to be features of nature and not simply creations of theory without correlates in the world. The chances that several unaffiliated theories will cooperate to manufacture artifactual evidence are less than that a single theory will do just that. (Kosso 1992, 155/156)

Kosso vertritt die Ansicht, dass die Unabhängigkeit der Theorien einen Einfluss auf die Wahrscheinlichkeit hat, mit der Beobachtungen falsche Annahmen in Theorien widerlegen. Eine Theorie, die nicht wirklich existierende Gegenstände und Ereignisse postuliert, soll mit größerer Wahrscheinlichkeit empirisch widerlegt werden, wenn die Beobachtung unabhängig theoriebeladen ist, als wenn sie selbstabhängig beladen ist.

Um eine Bewertung dieser Annahme zu ermöglichen, möchte ich eine formale Schreibweise für die Wahrscheinlichkeiten der Widerlegung und Bestätigung von Theorien einführen, von denen auch in Kossos Zitat die Rede ist. Hierbei sind allerdings keine subjektiven Wahrscheinlichkeiten etwa im Sinne des Bayesianismus gemeint. Vielmehr scheint es am plausibelsten, die Wahrscheinlichkeiten als objektive Wahrscheinlichkeiten der wissenschaftlichen Verfahren der empirischen Prüfung von Theorien aufzufassen. Demnach meint man mit „Wahrscheinlichkeit der Widerlegung einer weitgehend falschen Theorie durch unabhängige Beobachtungen" die objektive Wahrscheinlichkeit (Häufigkeit oder Neigung) des Prozesses der empirischen Prüfung von Theorien, für überwiegend falsche und unabhängig geprüfte Theorien ein insgesamt widerlegendes Ergebnis zu erbringen. ${ }^{17}$

17 Vgl. Mayo (1996) für eine Ausarbeitung der Idee, dass es objektive Wahrscheinlichkeiten der Widerlegung und Bestätigung von Hypothesen durch eine Prüfung gibt. 
Hier und im Folgenden sollen $W$ für „,die geprüfte Theorie ist überwiegend wahr“, Wid für „Beobachtungen widerlegen die geprüfte Theorie“, Best für „Beobachtungen bestätigen die geprüfte Theorie“ und $U$ für „die Beobachtungen sind überwiegend unabhängig theoriebeladen" stehen. $\sim W$ steht dementsprechend für ,die geprüfte Theorie ist nicht überwiegend wahr, d.h. sie ist in weiten Teilen falsch“, $S$ steht für ,die Beobachtungen sind überwiegend selbstabhängig theoriebeladen“. Es soll zudem angenommen werden, dass die Prüfung durch eine große Anzahl von Beobachtungen immer zu einem zumindest vorläufigen Ergebnis, d.h. zu einer Widerlegung oder einer Bestätigung führt.

Kossos Behauptung lässt sich dann als die Annahme fassen, dass die Wahrscheinlichkeit einer Widerlegung durch Beobachtungen, gegeben die geprüfte Theorie ist in weiten Teilen falsch und die Beobachtungen sind überwiegend unabhängig theoriebeladen, im Allgemeinen höher ist als die Wahrscheinlichkeit einer Widerlegung, gegeben eine falsche geprüfte Theorie und selbstabhängig theoriebeladene Beobachtungen:

(Wid-falsch)

$$
P(\text { Wid / } \sim W \& U)>P(W i d / \sim W \& S)
$$

Wie sich gleich zeigen wird, lassen sich für diese Annahme gute Gründe angeben. Doch dann sind mindestens zwei weitere Punkte zu untersuchen: (1) Sind in den Wissenschaften typischerweise die beladenden Theorien von den zu prüfenden Theorien unabhängig, oder könnten dies sein? (2) Lässt sich durch (Wid-falsch) begründen, dass unabhängig theoriebeladene Beobachtungen eine objektive Prüfung von Theorien bzw. eine objektivere Prüfung als selbstabhängig theoriebeladene Beobachtungen erlauben?

\section{b) Sind Beobachtungen typischerweise unabhängig theoriebeladen?}

Eine Behandlung des ersten Punkts setzt Klarheit darüber voraus, welche Formen der Theoriebeladenheit genau bestehen. ${ }^{18}$ Bei einigen Versionen der Theoriebeladenheit ist eine Unabhängigkeit plausiblerweise möglich. So können astronomische Beobachtungen von optischen Theorien abhängen, etwa indem sie die Funktion der verwendeten Instrumente oder die optischen Störungen durch die Erdatmosphäre erklären. Doch mit Hilfe solcher Beobachtungen werden keine optischen, sondern astrophysikalische Theorien überprüft. Wenn jedoch die Funktion oder Störungsanfälligkeit eines Strommessgeräts erklärt werden muss, werden ziemlich genau jene elektromagneti-

18 Vgl. Kosso (1989), Kap. 3, für Fallstudien zur Theorieabhängigkeit von Beobachtungen und Kosso (1992), 114-119, für Überlegungen zu verschiedenen Formen der Theoriebeladenheit. 
schen Theorien herangezogen werden müssen, die zu prüfen eine zentrale Funktion des Geräts ist. Es wäre nicht erstaunlich, wenn sich herausstellen sollte, dass häufig zumindest ein Teil der Erklärung der Gerätefunktion auf die Theorie angewiesen ist, die das untersuchte Phänomen beschreibt. Denn schließlich müssen Geräte, um überhaupt in einer Untersuchung dienen zu können, kausalen Kontakt zum Phänomen haben, die Theorie des Instruments muss daher typischerweise auch auf kausale Eigenschaften des Phänomens rekurrieren. Die geprüfte Theorie ist aber gerade für solche Eigenschaften zuständig.

Auch andere Varianten der Theoriebeladenheit führen zu Selbstabhängigkeit. So wurde von Feyerabend behauptet, dass eine empirisch geprüfte Theorie notwendigerweise die für sie relevanten Beobachtungssätze belade, da einerseits die Beobachtungssätze nur für die Theorie relevant sein könnten, wenn es evidenzielle bzw. inferenzielle Relationen zwischen den Beobachtungssätzen und der Theorie gebe, andererseits diese inferenziellen Relationen aber für die Bedeutung der Beobachtungssätze konstitutiv seien. ${ }^{19}$ Es bestehen dann aber zumindest vorläufige Zweifel daran, dass Beobachtungen typischerweise von anderen Theorien beladen werden als denen, die sie prüfen. Genauere Auskunft bekommt man nur, indem man die Varianten von Theoriebeladenheit untersucht.

\section{c) Unabhängigkeit und Objektivität I: Einzelne Theorien}

Aber selbst wenn unabhängig theoriebeladene Beobachtungen typischerweise möglich wären, stellt sich die zweite oben angegebene Frage: Stärkt im Allgemeinen die Unabhängigkeit der beladenden Theorie die Objektivität der Prüfung? Hierbei muss man zwei Fälle unterscheiden. Im einen Fall, der in diesem Unterabschnitt besprochen werden soll, wird eine einzelne Theorie durch unabhängig theoriebeladene Beobachtungen geprüft. ${ }^{20} \mathrm{Im}$ zweiten Fall wird eine ganze Reihe bzw. ein Netz, von Theorien durch Beobachtungen geprüft, wobei typischerweise die Beobachtungen, die jeweils eine Theorie

19 Siehe Feyerabend (1958).

20 Mit der Annahme dieses Falls soll nicht bestritten werden, dass für die Ableitung von Beobachtungsresultaten aus einer Theorie typischerweise andere Theorien als Hilfsannahmen hinzugezogen werden müssen. Häufig schließt man zwar aus diesem Umstand, dass im strikten Sinn nur eine Menge von Theorien, nicht aber einzelne Theorien empirisch geprüft werden können. Für diesen Fall soll aber davon ausgegangen werden, dass die Hilfsannahmen, die für die Ableitung bzw. Vorhersage benötigt werden, als unproblematisch gelten können und daher das Beobachtungsresultat einer einzelnen Theorie zurechenbar ist. 
prüfen, von anderen Theorien desselben Netzes beladen sind. Diesen Fall untersuche ich im nächsten Unterabschnitt.

Kossos obige Behauptung (Wid-falsch) kann man für die beiden Fälle jeweils unterschiedlich verstehen. Im Fall der Prüfung einer einzelnen Theorie wird behauptet, dass unabhängig theoriebeladene Beobachtungen die Falschheit einer Theorie mit größerer Wahrscheinlichkeit empirisch aufdecken als selbstabhängig beladene Beobachtungen. Diese Behauptung ist für sich genommen recht plausibel. Denn man kann zunächst davon ausgehen, dass völlig unabhängig von der Wahrheit oder Falschheit einer zu prüfenden Theorie die Wahrscheinlichkeit, dass Beobachtungen einer Theorie widersprechen, bei unabhängig theoriebeladenen Beobachtungen größer ist als bei selbstabhängig beladenen Beobachtungen. Daher ist für eine Theorie die Wahrscheinlichkeit einer Widerlegung durch unabhängige Beobachtungen höher als durch selbstabhängige Beobachtungen:

$$
P(\text { Wid / U })>P(\text { Wid / S). }
$$

Denn wenn eine Theorie eine Beobachtung beeinflusst, macht dies wahrscheinlicher, dass die Beobachtung mit dieser Theorie vereinbar ist, also sie bestätigt bzw. nicht widerlegt. Eine Beeinflussung durch eine unabhängige Theorie macht dagegen eine Verträglichkeit weniger wahrscheinlich und lässt daher ein widerlegendes Ergebnis erwarten. Aber (Wid-falsch) ist dann auch plausibel. Denn es ist auch von einer falschen Theorie wahrscheinlicher, dass sie ihre eigene Basis auf eine für sie günstige Weise beeinflusst und so eine empirische Widerlegung verhindert, als dass eine unabhängige Theorie die Beobachtung so beeinflusst.

Allerdings folgt hieraus nicht schon, dass unabhängige Beobachtungen objektiver als selbstabhängige sind. Denn empirische Prüfungen haben nicht nur eine kritische Rolle, indem sie Theorien, die in weiten Teilen falsch sind, empirisch zurückweisen müssen. Sie haben auch eine konstruktive Aufgabe: Sie sollten weitgehend wahre Theorien bestätigen. Hierbei scheinen aber selbstabhängige Beobachtungen besser abzuschneiden als unabhängige. Denn unabhängige Beobachtungen widerlegen nicht nur falsche Theorien mit höherer Wahrscheinlichkeit als selbstabhängige Beobachtungen. Es scheint auch wahrscheinlicher, dass eine wabre Theorie durch unabhängige Beobachtungen eher widerlegt wird als durch selbstabhängige. Denn zum einen beeinflusst bei einer selbstabhängigen Prüfung eine Theorie ihre eigene Basis, und dies macht eine Widerlegung unwahrscheinlicher. Zum anderen ist die geprüfte Theorie ja der Annahme nach wahr, wohingegen über die Wahrheit oder Falschheit der unabhängigen Theorien bisher nichts gesagt ist. Beobachtungen, die von wahren Theorien beeinflusst werden, sind aber im Allgemeinen 
zuverlässiger als Beobachtungen, die von irgendwelchen - zum Teil wahren, zum Teil falschen Theorien - beeinflusst sind. Selbstabhängige Beobachtungen werden daher im Allgemeinen eine wahre Theorie eher bestätigen als unabhängige Beobachtungen:

(Best-wahr)

$$
P(\text { Best / W\&U })<P(\text { Best / W\&S }) \text {. }
$$

Damit ist aber unklar, was sich aus der Unabhängigkeit für die Objektivität von Beobachtungen ergibt. Schon die erste Behauptung Kossos, dass unabhängige Beobachtungen objektiver als selbstabhängige sind, wird fragwürdig. Die Zielvorgabe für Objektivität sieht vor, dass die Bestätigung einer systematisch tugendhaften Theorie durch objektive Beobachtungen die überwiegende Wahrheit der Theorie wahrscheinlich macht. Unabhängigkeit würde nur dann im Vergleich zu Selbstabhängigkeit zu größerer Objektivität führen, wenn bei Unabhängigkeit die Wahrscheinlichkeit der Wahrheit einer bestätigten Theorie höher wäre, also wenn

(Unab-objektiver) $P(W /$ Best\& $U)>P(W /$ Best\&S $)$.

Für die Wahrscheinlichkeit der Wahrheit einer Theorie gegeben ihre Bestätigung gilt nun:

(W/Best)

$$
P(W / \text { Best })=\frac{1}{\frac{P(\text { Best } / \sim W) \cdot P(\sim W)}{P(\text { Best } / W) \cdot P(W)}+1} \cdot{ }^{21}
$$

Unabhängigkeit führt daher zu größerer Objektivität als Selbstabhängigkeit, d.h. (Unab-objektiver) gilt genau dann, wenn

$$
\frac{P(\text { Best } / \sim W \& U) \cdot P(\sim W / U)}{P(\text { Best } / W \& U) \cdot P(W / U)}<\frac{P(\text { Best } / \sim W \& S) \cdot P(\sim W / S)}{P(\text { Best } / W \& S) \cdot P(W / S)} .
$$

Diese Bedingung lässt sich noch vereinfachen. Denn da die Wahrscheinlichkeit der Wahrheit einer zur Prüfung kommenden Theorie klarerweise unabhängig davon ist, ob diese Prüfung mit selbstabhängig oder unabhängig theoriebeladenen Beobachtungen durchgeführt wird, ist $\mathrm{P}(\sim \mathrm{W} / \mathrm{U}) / \mathrm{P}(\mathrm{W} / \mathrm{U})$ $=\mathrm{P}(\sim \mathrm{W} / \mathrm{S}) / \mathrm{P}(\mathrm{W} / \mathrm{S})$.

${ }^{21}$ Es ist: $P(W /$ Best $)=\frac{P(W \& \text { Best })}{P(\text { Best })}=\frac{P(\text { Best } / W) \cdot P(W)}{P(\text { Best } / W) \cdot P(W)+P(\text { Best } / \sim W) \cdot P(\sim W)}$

$$
=\frac{1}{\frac{P(\text { Best } / \sim W) \cdot P(\sim W)}{P(\text { Best } / W) \cdot P(W)}+1} .
$$


(Unab-objektiver) gilt daher genau dann, wenn

$$
\frac{P(\text { Best } / \sim W \& U)}{P(\text { Best } / W \& U)}<\frac{P(\text { Best } / \sim W \& S)}{P(\text { Best } / W \& S)} .
$$

Hieran lässt sich nun zeigen, welchen Einfluss die Unabhängigkeit der beladenden Theorie bei der Prüfung einzelner Theorien auf die Objektivität der Prüfung hat. Es gilt zwar einerseits

(Wid-falsch)

$$
\begin{aligned}
& P(\text { Wid / } \sim W \& U)>P(W i d / \sim W \& S), \text { d.h. } \\
& P(\text { Best / } \sim W \& U)<P(\text { Best / } \sim W \& S) .
\end{aligned}
$$

Damit ist der linke Zähler kleiner als der rechte, was die Objektivität unabhängiger Beobachtungen fördert. Aber (Best-wahr) beinhaltet, dass

(Best-wahr)

$$
P(\text { Best } / W \& U)<P(\text { Best } / W \& S),
$$

d.h. der rechte Nenner ist größer als der linke, was der größeren Objektivität unabhängiger Beobachtungen entgegensteht. Das bedeutet, dass man keinen Grund für die Annahme hat, dass die Unabhängigkeit der beladenden Theorie im Allgemeinen die Objektivität von Prüfungen einzelner Theorien erhöht, auch wenn man wie Kosso von (Wid-falsch) ausgeht.

Damit ist aber noch nicht gesagt, dass unabhängige Beobachtungen unter keinen Umständen objektiver als selbstabhängige sind. Denn die Wahrscheinlichkeit, mit der unabhängige Beobachtungen überwiegend wahre geprüfte Theorien bestätigen, hängt plausiblerweise auch davon ab, ob die unabhängig beladenden Theorien überwiegend wahr oder in weiten Teilen falsch sind. Wenn unabhängige, aber wahre Theorien den Beobachtungsprozess beeinflussen, wird das Ergebnis für eine überwiegend wahre geprüfte Theorie mit geringerer Wahrscheinlichkeit widerlegend sein, als wenn falsche unabhängige Theorien den Prozess beeinflussen. Denn man kann erwarten, dass die Verlässlichkeit des Beobachtungsprozesses bei Beeinflussung durch wahre Theorien höher ist als bei Beeinflussung durch falsche Theorien.

Dies bedeutet aber, dass der Unterschied, den Selbstabhängigkeit oder Unabhängigkeit für die Wahrscheinlichkeit der Bestätigung einer wahren zu prüfenden Theorie machen, möglicherweise dann nur minimal ist, falls die unabhängigen Theorien überwiegend wahr sind. In einem solchen Fall würde also die Ungleichung (Best-wahr) kaum ins Gewicht fallen, wohingegen die Ungleichung (Wid-falsch) mindestens unverändert bestehen bleibt. Dies lässt erwarten, dass unabhängige Beobachtungen jedenfalls dann objektiver sind, wenn die unabhängig beladenden Theorien überwiegend wahr sind. Allerdings gilt für den Fall, dass die unabhängigen Theorien weitgehend falsch sind, gerade das Gegenteil. Denn hier verstärkt sich noch die Ungleichheit in 
(Best-wahr), und in diesem Fall sind selbstabhängige Beobachtungen objektiver.

Damit zeigt sich, dass Unabhängigkeit nur dann dabei hilft, die erhöhte Objektivität von Prüfungen einzelner Theorien zu begründen, wenn man bereits auf andere Theorien zurückgreifen kann, die wahrscheinlich überwiegend wahr sind. Ein Optimismus lässt sich dann möglicherweise gegenüber unabhängig geprüften und bestätigten Theorien begründen, sofern man ihn schon für die unabhängigen, beladenden Theorien begründet hat. Aber eine Möglichkeit, den Optimismus gegenüber einzelnen, geprüften Theorien ohne anderweitige optimistische Voraussetzungen zu begründen, bietet sich durch das Merkmal Unabhängigkeit nicht.

\section{d) Unabhängigkeit und Objektivität II: Theoriennetze}

Das eben festgestellte Problem für die Begründung des Optimismus durch die unabhängig theoriebeladene Prüfung einzelner Theorien ergab sich dann, wenn man nichts über den Status der unabhängigen Theorien voraussetzen kann. Die Annahme, dass die unabhängigen, beladenden Theorien selbst überwiegend wahr sind, setzt aber gegenüber diesen Theorien schon voraus, was man für die einzelnen, geprüften Theorien erst zeigen möchte. Stattdessen könnte man aber auch annehmen, dass die unabhängigen Theorien selbst empirisch bestätigt sind - bestätigt in derselben Weise, in der die einzelne, einzuschätzende Theorie es ist. Dies führt zur Idee, dass die Einheit, der gegenüber ein Optimismus zu begründen ist, letztlich nicht eine einzelne Theorie, sondern ein ganzes Netz von Theorien ist. Ein Optimismus würde dann beinhalten, dass die meisten Theorien dieses Netzes überwiegend wahr sind, gegeben sie sind empirisch bestätigt. Theorien dieses Netzes werden dann unabhängig geprüft, wenn jeweils andere Theorien desselben Netzes die Prüfung beladen. Kann diese Form der empirischen Prüfung den Optimismus begründen?

Die erste Kosso'sche Behauptung, dass Unabhängigkeit die Objektivität erhöht, könnte sich im Fall der unabhängigen Prüfung eines Theoriennetzes auf die folgenden, plausiblen Annahmen zu Widerlegungs- und Bestätigungswahrscheinlichkeiten stützen: Erstens ist es sehr unwahrscheinlich, dass ein Netz solcher Theorien insgesamt durch unabhängig beladene Beobachtungen bestätigt wird, obwohl die meisten dieser Theorien überwiegend falsch sind. Denn um eine solche irreführende Bestätigung zustande zu bringen, müssten falsche Theorien häufig die Beobachtungen in solcher Weise beeinflussen, dass sie für andere falsche Theorien günstig ausfallen. Dies ist aber bei Unabhängigkeit der Theorien unwahrscheinlich, und unwahrscheinlicher als bei selbstabhängiger Beladung. Es wäre also: 
(Wid-falsch-Netz)

$$
P(\text { Wid } / \sim W \& U)=\text { groß, und } P(\text { Wid } / \sim W \& U)>P(W i d / \sim W \& S) \text {, }
$$

d.h. $P($ Best $/ \sim W \& U)=$ klein, und $P($ Best $/ \sim W \& U)<P($ Best $/ \sim W \& S)$.

( $W$ steht hier für „die meisten Theorien des Netzes sind überwiegend wahr“, $\sim W$ heißt dementsprechend, ,viele Theorien des Netzes sind in weiten Teilen falsch“.) Zweitens wäre von einem Theoriennetz, das zumeist aus überwiegend wahren Theorien besteht, bei Unabhängigkeit aber wahrscheinlich, dass es empirisch bestätigt wird. Schließlich würden überwiegend wahre, wenn auch unabhängige Theorien die Beobachtungen beladen. Dies erhöht deren Verlässlichkeit und macht die Bestätigung wahrer Theorien wahrscheinlich. Anders als bei der Prüfung einzelner Theorien ist bei der Prüfung eines Netzes von Theorien also die Wahrscheinlichkeit der Bestätigung einer wahren Theorie bei Unabhängigkeit nicht klein, sondern eher groß, und der Unterschied zu selbstabhängigen Prüfungen fällt somit gering aus:

(Best-wahr-Netz)

$$
P(\text { Best } / W \& U)=\text { groß, und } P(\text { Best } / W \& U) \approx P(\text { Best } / W \& S) .
$$

Dann sind aber unabhängige Prüfungen von Theoriennetzen objektiver als selbstabhängige. Denn für die Prüfung von Theoriennetzen gilt:

$$
\frac{P(\text { Best } / \sim W \& U)}{P(\text { Best } / W \& U)}<\frac{P(\text { Best } / \sim W \& S)}{P(\text { Best } / W \& S)}, \text { d.h. }
$$

(Unab-objektiver) $P(W /$ Best\&U $)>P(W /$ Best\&S).

Bedeutet dies auch, dass Beobachtungen, die insgesamt die empirische Basis für ein ganzes Netz von Theorien darstellen und durch Theorien aus diesem Netz unabhängig beladen sind, nicht nur im Vergleich zu selbstabhängigen Beobachtungen objektiver, sondern auch absolut objektiv sind? Hierzu müsste es von einem solchen Netz von Theorien, das solcherart empirisch geprüft und hierbei betätigt wurde, wahrscheinlich sein, dass es überwiegend wahr ist. Es müsste also gelten:

(Unab-objektiv) $P(W /$ Best\&U $)=$ groß.

Allerdings folgt dies nicht aus (Wid-falsch-Netz) und (Best-wahr-Netz). Dies kann man sehen, wenn man nochmals die obige Gleichung (W/Best) heranzieht und für diesen Fall auf $U$ bedingt:

$\left(\mathrm{W} /\right.$ Best\&U) $\quad P(W /$ Best \& $U)=\frac{1}{\frac{P(\text { Best } / \sim W \& U) \cdot P(\sim W / U)}{P(\text { Best } / W \& U) \cdot P(W / U)}+1}$. 
Nun besagen (Wid-falsch-Netz) und (Best-wahr-Netz) zwar, dass $P($ Best $/ \sim W \& U)$ klein und $P(B e s t / W \& U)$ groß sind. Damit aber der Nenner auch insgesamt klein und damit die Wahrscheinlichkeit der Wahrheit eines unabhängig bestätigten Theoriennetzes groß ist, dürfen nicht $P(\sim W / U)$ groß und $P(W / U)$ klein sein. $P(\sim W / U)$ und $P(W / U)$ stehen für die Wahrscheinlichkeit von zur Prüfung kommenden Theoriennetzen, in weiten Teilen falsch bzw. überwiegend wahr zu sein.

Angenommen, bei Unabhängigkeit ist die Bestätigungswahrscheinlichkeit für in weiten Teilen falsche Theoriennetze mit $P($ Best $/ \sim W \& U)=0,2$ niedrig; die Bestätigungswahrscheinlichkeit für wahre Theoriennetze $P(B e s t / W \& U)$ ist dagegen 0,8 . Wenn aber die Wahrscheinlichkeit, dass die zur Prüfung gestellten Theoriennetze überwiegend falsch sind, mit $P(\sim W / U)=0,9$ recht hoch ist, während die Wahrscheinlichkeit, dass ein weitgehend wahres Theoriennetz zur Prüfung gestellt wird, mit $P(W / U)=0,1$ niedrig ist, ergibt sich dennoch für $P(W /$ Best\& $U)$ nur ungefähr 0,3 . In einem solchen Fall wäre es trotz der Geltung von (Wid-falsch-Netz) und (Best-wahr-Netz) von einem Netz bestätigter Theorien unwahrscheinlich, dass die meisten Theorien des Netzes überwiegend wahr sind. Der Schluss von der Bestätigung der Theorien auf ihre überwiegende Wahrheit wäre nicht gültig.

Dieses Zahlenbeispiel zeigt ein Hindernis für den Nachweis der Objektivität von Beobachtungen bei unabhängiger Theoriebeladung auf. Auch wenn wahre Theoriennetze wahrscheinlich bestätigt und falsche wahrscheinlich widerlegt werden, hieße es die ,base rate fallacy ${ }^{`}$ zu begehen, daraus auf die wahrscheinliche Wahrheit bestätigter Theorien zu schließen. Vielleicht sind falsche Theoriennetze als solche sehr viel wahrscheinlicher als wahre. Dann sind bestätigte Theorien nicht wahrscheinlich wahr.

$\mathrm{Zu}$ beachten ist hierbei erstens, dass $P(\sim W / U)$ und $P(W / U)$ die Wahrscheinlichkeit der Falschheit bzw. Wahrheit von Theoriennetzen vor aller empirischen Prüfung bezeichnen. Man muss in der Auswahl dieser Menge von Vorschlägen auch solche Netze bzw. Theorien in den Netzen einschließen, die wegen offensichtlicher empirischer Inadäquatheit gar nicht erst einer tatsächlichen ausführlichen Prüfung unterzogen werden. Denn der Ausschluss solcher Theorien geschieht bereits auf der Grundlage von Beobachtungen. Der gegenwärtig diskutierte Vorschlag basiert die Objektivität von Beobachtungen aber allein darauf, dass sie als unabhängig beladen in der Prüfung von Theoriennetzen eingesetzt werden. Auch der Gebrauch von Beobachtungswissen in einer Vorauswahl der Theorien und Netze, die überhaupt zu einer tatsächlichen Prüfung gelangen, muss man in diesem Zusammenhang daher als eigentlich schon empirische Prüfung rekonstruieren. Doch dies legt nahe, dass die Wahrscheinlichkeit recht hoch ist, dass 
solchermaßen vorgeschlagene und zur Prüfung gestellte Theoriennetze in weiten Teilen falsch sind.

Zweitens ist dem Vorschlag zufolge ein ganzes Theoriennetz die Einheit der empirischen Prüfung. Der Unterschied zum vorigen Unterabschnitt besteht gerade darin, dass nicht einzelne Theorien empirisch bestätigt werden, um erst darauf aufbauend andere Theorien zu prüfen. Der Vorteil unabhängiger Beobachtungen gegenüber selbstabhängigen Beobachtungen ergibt sich erst, indem man ein holistisches Vorgehen gegenüber einem schrittweisen bevorzugt. Doch auch dadurch wird die Wahrscheinlichkeit erhöht, dass in weiten Teilen falsche Theorien zur Prüfung gestellt werden. Denn je inhaltsreicher eine Theorie bzw. ein Netz unabhängiger Theorien ist, desto unwahrscheinlicher ist es, dass man - vor aller Berücksichtigung empirischer Befunde - gerade überwiegend die Wahrheit trifft.

Ein Theoriennetz kann also durch Beobachtungen, die durch unabhängige Theorien dieses Netzes beladen sind, zwar besser empirisch geprüft werden als durch selbstabhängige Beobachtungen. Die erste, komparative These Kossos konnte daher für diesen Fall begründet werden. Aber allein die Unabhängigkeit der beladenden von den jeweils geprüften Theorien reicht nicht aus, um die Objektivität der Beobachtungen als solche zu sichern. Die zweite These Kossos, dass unabhängige Beobachtungen absolut gesehen objektiv sind, konnte daher für den Fall der Prüfung von Theoriennetzen nicht begründet werden. Noch dürftiger fällt das Ergebnis für den Fall der Prüfung einzelner Theorien aus. Hier hat die Untersuchung gar keine Gründe dafür ergeben, dass Unabhängigkeit im Allgemeinen epistemische Vorteile mit sich bringt. Solche Vorteile bestehen vielmehr nur unter der Voraussetzung, dass man schon über unabhängige Theorien verfügt, die selbst optimistisch einzuschätzen sind. Die unabhängige Beladung von Beobachtungen hat sich damit in diesen Fall lediglich als ein Vehikel erwiesen, um Optimismus von einer Theorie auf eine andere zu transferieren. ${ }^{22}$ Die Frage, warum Beobachtungen trotz ihrer Theoriebeladenheit objektiv sind, bleibt offen. Die Hinweise auf die Effektivität und die unabhängige Beladung der Beobachtungen können bestenfalls einen Teil einer detaillierten Antwort ausmachen. Für diese detaillierte Antwort sind verschiedene Arten von Beobachtungen zu unterscheiden und deren mögliche Theoriebeladenheit im Einzelnen zu diskutieren.

22 Dies bedeutet nicht, dass der neue Optimismus in eine Theorie dann nur in der Kohärenz oder in anderen, rein systematischen Beziehungen zur anderen, schon optimistisch einzuschätzenden Theorien begründet liegt. Der neue Optimismus wird vielmehr dadurch gefördert, dass die unabhängige, optimistisch einzuschätzende Theorie es erlaubt, die neue Theorie objektiv (bzw. objektiver) empirisch zu prüfen. 


\section{Zusammenfassung}

In diesem Kapitel habe ich zwei mögliche Merkmale theoriebeladener Beobachtungen diskutiert, Effektivität und Unabhängigkeit. Beide Merkmale werden in der Regel gegen das Bedenken angeführt, dass Theoriebeladenheit zu einer viziösen Zirkularität in der empirischen Prüfung von Theorien führt.

Zunächst habe ich das Merkmal der Effektivität analysiert (Abschn. 2). Die verbreitete Ansicht, dass die Effektivität von Beobachtungen daran hängt, ob die geprüften Theorien einem Fehlschlagsrisiko ausgesetzt werden, erwies sich als zu eng. Auch die Annahme, dass sich die Ineffektivität einer theoriebeladenen Beobachtung letztlich immer als prädiktive Irrelevanz der Hypothese für ein zugrunde liegendes, theorieunabhängiges Beobachtungsergebnis rekonstruieren lässt, habe ich zurückgewiesen. Stattdessen hat sich gezeigt, dass eine Beobachtung trotz ihrer Theoriebeladenheit für eine Theorie effektiv ist, wenn die Beobachtung, soweit sie mit der Theorie übereinstimmt oder ihr widerspricht, auch empirisch bestimmt ist (Abschn. 2b). Die Effektivität von Beobachtungen ist daher eine minimale, aber bei weitem nicht hinreichende Bedingung für die Objektivität theoriebeladener Beobachtungen (Abschn. 2c).

Wenn bei Beobachtungen die beladenden Theorien unabhängig von den geprüften Theorien sind, muss man zwei Fälle unterscheiden. Wenn man die Prüfung einer einzelnen Theorie betrachtet, ergeben sich aus der unabhängigen Beladung keine generellen epistemischen Vorteile. Diese hängen vielmehr daran, wie wahrscheinlich es von der unabhängigen Theorie ist, dass sie selbst wahr ist (Abschn. 3c). Wenn man dagegen die Prüfung eines ganzen Netzes unabhängiger Theorien betrachtet, erweisen sich unabhängig geprüfte und bestätigte Theorien als mit höherer Wahrscheinlichkeit wahr als selbstabhängig geprüfte und bestätigte Theorien. Über diese komparative These hinaus lässt sich aber die Objektivität theoriebeladener Beobachtungen als solcher auch in diesem Fall nicht begründen (Abschn. 3d). Es zeigt sich, dass der Hinweis auf die abstrakten Merkmale Effektivität und Unabhängigkeit nur einen Teil einer detaillierten Begründung der Objektivität theoriebeladener Beobachtungen ausmachen kann.

\section{Anhang zu Abschnitt 2b}

Wenn man die Jeffrey-Regel für Konditionalisierung

$$
P^{\prime}(H)=P(H / O) \cdot P^{\prime}(O)+P(H / \sim O) \cdot P^{\prime}(\sim O)
$$

voraussetzt, gilt: 
Ein empirischer Test ist ineffektiv, d.h. $P^{\prime}(H)=P(H)$, g.d.w. er nicht-empirisch ist, d.h. $P^{\prime}(O)=P(O)$, oder $H$ prädiktiv irrelevant ist, d.h. $P(O / H)=$ $P(H)$.

Beweis:

(Jeffrey-Kond) $P^{\prime}(H)=P(H / O) \cdot P^{\prime}(O)+P(H / \sim O) \cdot P^{\prime}(\sim O)$

$$
\Leftrightarrow \quad P^{\prime}(H)=P(H / O) \cdot P^{\prime}(O)+P(H / \sim O) \cdot\left[1-P^{\prime}(O)\right]
$$

(i) $\Leftrightarrow P^{\prime}(H)=P^{\prime}(O) \cdot[P(H / O)-P(H / \sim O)]+P(H / \sim O)$;

zudem ist ein Theorem des Wahrscheinlichkeitskalküls:

$$
P(H)=P(H / O) \cdot P(O)+P(H / \sim O) \cdot P(\sim O)
$$

(ii) $\Leftrightarrow P(H)=P(O) \cdot[P(H / O)-P(H / \sim O)]+P(H / \sim O)$

aus (i) und (ii) folgt:

(Ineff.)

$$
\begin{array}{ll} 
& P^{\prime}(H)=P(H) \\
\Leftrightarrow \quad & P^{\prime}(O) \cdot[P(H / O)-P(H / \sim O)]+P(H / \sim O) \\
& =P(O) \cdot[P(H / O)-P(H / \sim O)]+P(H / \sim O) \\
\Leftrightarrow \quad & P^{\prime}(O)=P(O) \text { oder } P(H / O)=P(H / \sim O) \\
\Leftrightarrow \quad & P^{\prime}(O)=P(O) \text { oder } P(O / H)=P(O) \text { (mit Bayes' Theorem). }
\end{array}
$$

